Radiologe 2021 · 61:123-125

https://doi.org/10.1007/s00117-021-00804-8

Angenommen: 4. Januar 2021

(c) Springer Medizin Verlag GmbH, ein Teil von Springer Nature 2021

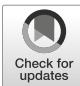

\author{
R. Schulz-Wendtland' $\cdot$ M. Reiser ${ }^{2}$ \\ ${ }^{1}$ Radiologisches Institut, Gynäkologische Radiologie, Universitätsklinikum Erlangen, Erlangen, \\ Deutschland \\ ${ }^{2}$ Klinik und Poliklinik für Radiologie, LMU-Klinikum, München, Deutschland
}

\section{Mammadiagnostik: von der klinischen Diagnostik bis zur künstlichen Intelligenz}

Forschungsbedarf? Wie kann die Bildgebung (das meint natürlich die Menschen, die sich damit beschäftigen), dazu beitragen, dass Früherkennung, Patientenmanagement und letztlich die Behandlungsergebnisse weiter verbessert werden und damit die Letalität von Brustkrebs gesenkt wird?“

Dabei fangen wir keineswegs bei null an! Im Gegenteil: Es wurden eindrucksvolle Erfolge erzielt. Heindel et al. setzen sich kritisch mit der Frage auseinander, was durch das vor 10 Jahren eingeführte deutsche MammographieScreening-Programm erreicht wurde. Eine zentrale Rolle nimmt dabei eine rigorose Qualitätssicherung ein. Als ein wichtiger Erfolg des Screenings ist zu werten, dass die Inzidenzrate fortgeschrittener Mammakarzinome deutlich gesenkt wurde. Daher kann eine Senkung der Brustkrebsmortalität durch regelmäßiges Screening als wahrscheinlich angesehen werden. Das deutsche Mammographie-Screening-Programm kann insgesamt als großer Erfolg angesehen werden, wenngleich abschließende Ergebnisse hinsichtlich der Reduktion der Letalität noch ausstehen. Gleichzeitig werden Studien zur evidenzbasierten Weiterentwicklung des MammographieScreening-Programms durchgeführt, wie die Einbeziehung älterer Frauen bis 75 Jahren, die Kombination von digitaler Brust-Tomosynthese und "full-field digital mammography“ sowie der additive Ultraschall und die additive Magnetresonanztomographie (MRT) bei Frauen mit dichter Brust.
In dem Beitrag von Müller-Schimpfle et al. wird die senologische Diagnostik handelt. Die Autoren weisen darauf hin, dass seit der Jahrtausendwende substanzielle Verbesserungen in der Diagnostik und Therapie des Mammakarzinoms erreicht wurden. Dazu haben nicht zuletzt das Zertifizierungsprogramm für Brustzentren und die Verabschiedung von Leitlinien der Stufe 3 beigetragen. Inzwischen werden $77 \%$ der in Deutschland diagnostizierten Mammakarzinome in zertifizierten Brustzentren behandelt, in denen nach definierten Qualitätskriterien und leitliniengerecht vorgegangen wird. Anhand von vier typischen Konstellationen, nämlich 1) risikobasierte Früherkennung, 2) stereotaktische Vakuumbiopsie im Screening-Programm, 3) Abklärungsdiagnostik bei symptomatischer Patientin und 4) Rezidivdiagnostik nach Mammakarzinom wird anschaulich und praxisnah das Vorgehen dargestellt und kritisch diskutiert.

Bick widmet sich in seinem Beitrag der intensivierten Brustkrebsfrüherkennung bei Hochrisikopatientinnen und macht deutlich, dass bei diesen Patientinnen der MRT eine Schlüsselrolle zukommt. Über $30 \%$ der Mammakarzinome werden primär nur in der MRT detektiert. Um eine möglichst gute Risiko-Nutzen-Relation $\mathrm{zu}$ erreichen, müssen die Eingangskriterien zu der intensivierten Brustkrebsfrüherkennung genau definiert werden. Neben dem Nachweis von Mutationen der Risikogene empfiehlt der Autor die Berechnung des 10-Jahres-Erkrankungsrisikos nach BOADICEA. in einem zertifizierten Brustzentrum be- 
Unverzichtbar für Screening-Programme und die Arbeit von zertifizierten Brustzentren sind die minimal-invasiven Mammainterventionen „MiMi“. Oft sind sie entscheidend für das weitere Patientenmanagement. Wie Hahn und Preibsch eindrucksvoll darlegen, bieten die MiMis die Möglichkeit, die relevanten Biomarker zu bestimmen, die für die Therapieentscheidungen maßgeblich sind.

Drei Beiträge dieses Themenheftes widmen sich interessanten methodischen Innovationen. Fallenberg berichtet über kontrastverstärkte Dual-Energybzw. spektrale Mammographie-Systeme, mit denen gleichzeitig mammographische Bilder generiert werden können und die Kontrastmittelanreicherung im Tumorgewebe, die auf der Neoangiogenese beruht, nachgewiesen werden kann. Die Ergebnisse dieses Verfahrens sind außerordentlich ermutigend, sodass die Autorin schlussfolgert, dass die kontrastverstärkte Mammographie eine wertvolle Ergänzung der bisher verfügbaren Methoden der Mammabildgebung sein kann.

Auch Wasner et al. widmen sich einem Thema, an dem schon seit Jahrzehnten gearbeitet wurde, bei dem aber erst in jüngster Vergangenheit richtungweisende Fortschritte gemacht wurden - der Fusion von Mammographie und Ultraschall. Schon lange ist die Kombination von Mammographie und Ultraschall in der Mammadiagnostik etabliert, da damit die Schwächen des einen Verfahrens durch die Stärken des anderen Verfahrens kompensiert werden können. Ein Problem ist häufig die Ortung und räumliche Einordnung von Befunden, die mit beiden Verfahren identifiziert werden. Durch die Fusion der Bilddaten aus Tomosynthese und automatisiertem 3-DUltraschall kann diese Problematik gelöst werden.

Weismann beschäftigt sich in seinem Beitrag mit der Sonoeleastographie der Mamma. Er geht von der bekannten klinischen Beobachtung aus, dass invasive Mammakarzinome im Allgemeinen härter sind als das normale Mammagewebe. Für die Sonoelastographie stehen verschiedene technische Verfahren zur Verfügung, die der Autor beschreibt und hinsichtlich ihrer diagnostischen Aussagekraft und ihrer Limitationen analysiert: 1. Strainelastographie, 2. Acoustic Radiation Force Impulse (ARFI) Imaging und ARFI mit Punkt-Scherwellenelastographie sowie 3. Scherwellenelastographie. Er kommt zu der Einschätzung, dass jede dieser Techniken die Spezifität des alleinigen B-Bild-Ultraschalls erhöht. Wenn allerdings das B-Bild höhergradig suspekt ist, darf aber auch dann nicht auf eine histologische Abklärung verzichtet werden, wenn die Sonoelastographie negativ ist.

Das übergreifende Paradigma der modernen Medizin ist das der Präzisionsmedizin, das darauf zielt „dem richtigen $\mathrm{Pa}$ tienten zum richtigen Zeitpunkt die richtige Therapie“ zugutekommen zu lassen. Um diesem Anspruch gerecht zu werden, nutzen DoGullo et al. die multiparametrische MRT, insbesondere die dynamische kontrastverstärkte MRT und die Diffusions-MRT und korrelieren diese mit Omics-Befunden (Genomics, Transcriptomics, Proteomics, Metabolomics), um aus den so generierten RadiogenomicsDaten tiefere Einblicke in die Tumorbiologie zu gewinnen. Die bisher vorliegenden Ergebnisse sprechen dafür, dass Radiomics eine hohe Treffsicherheit bei der Bestimmung des molekularen Subtyps und des Rezeptorstatus eines Mammakarzinoms erreicht und das Rezidivrisiko und das Therapieansprechen vorherzusagen vermag.

Nicht nur in der Medizin, sondern auch in vielen anderen Wissenschaftsgebieten und in der Technik werden die Verfahren der künstlichen Intelligenz (KI) nicht selten als seligmachender Heilbringer erwartet und begrüßt. Baltzer sieht in der Mammadiagnostik ein wichtiges und interessantes Anwendungsgebiet für die KI. Aus seiner Sicht sind bereits diverse Software-Tools verfügbar, die z.B. für die Screening-Mammographie brauchbar und nützlich sind. Defizite und damit erhebliches Entwicklungspotenzial erkennt er bei der Entwicklung von umfassenden Lösungen, mit denen die klinischen Abläufe unterstützt und fehlerresistent gemacht werden können. Er hofft, dass die Ärzte damit entlastet werden und die gewonnenen Freiräume für die Kommunikation mit den Pati- entinnen und den Fachkollegen genutzt werden können.

Wir hoffen, dass wir Ihnen, liebe Leserinnen und Leser, mit diesem Themenheft wertvolle und für Ihre tägliche Arbeit nützliche Informationen bieten. Den Autoren danken wir für ihr Engagement und dass sie ihre Expertise mit uns teilen.

Ihre

Rüdiger Schulz-Wendtland

Maximilian Reiser

\section{Korrespondenzadresse}

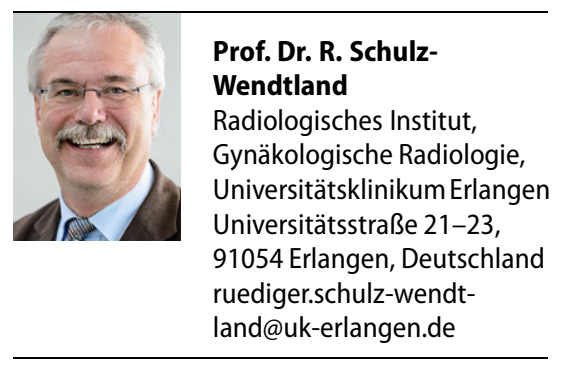

Interessenkonflikt. R. Schulz-Wendtland und M. Reiser geben an, dass kein Interessenkonflikt besteht. 
Hier steht eine Anzeige.

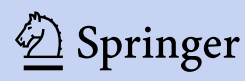

